\section{Response to: 'Infrapatellar fat pad resection during total knee replacement: yet another reason?' by Ryan}

We thank Ryan ${ }^{1}$ for the interest in our original paper $^{2}$ and response ${ }^{3}$ regarding to the associations between infrapatellar fat pad (IPFP) maximal area and knee osteoarthritic measures including symptoms and structural changes.

Our previous studies revealed that IPFP maximal area and volume were associated with reduced knee pain, decreased loss of cartilage volume and reduced risks of cartilage defect progression, indicating a beneficial effect of IPFP size. ${ }^{24}$ On the other hand, our further investigation demonstrated that IPFP signal intensity alteration was negatively associated with maximum area of IPFP and, moreover, associated with increased knee cartilage defects, subchondral bone marrow lesions and knee pain, suggesting IPFP with abnormal quality may play a detrimental role in knee osteoarthritis (OA). ${ }^{5}$

Based on these findings, we proposed that IPFP with normal qualities, rather than abnormal quality, should be preserved or not damaged during total knee arthroplasty (TKA), while IPFP with abnormal quality should be resected rather than preserved. Because of the biphasic effects of IPFP on OA, a proposed solution for improving patient outcomes after TKA involves using screening MRIs to identify IPFP signal intensity alteration, which is a subsequent indication for resection during TKA surgeries. ${ }^{3}$ This approach is not 'contrary' to approach offered by Sekiya, ${ }^{6}$ which suggested the use of postarthroplasty arthroscopic IPFP debridement. Moreover, the underlying rationales of two solutions are similar, despite different timing. Han $e t$ al $l^{5}$ also suggested that IPFP screening has the potential to reduce reoperation and perhaps reduce morbidity of severe knee pain post-TKA beyond $2 \%$.

Patellar tendon shortening could be one of the reasons for post-TKA anterior knee pain as findings from Gwyn et al's retrospective study ${ }^{7}$ reported that complete IPFP resection was associated with patellar tendon shortening 1 year after TKA. The authors had noted that studies also reporting IPFP excision during TKA did not alter the patellar tendon length. ${ }^{89}$ Despite the inconclusive effect of IPFP resection on patellar tendon shortening, the protective effect of normal IPFP on knee may be largely attributed to buffering and lubricating functions in knee joints, which absorb shocks from anterior knee and reduce friction between patellar tendon and tibia. ${ }^{10} 11$

The authors agree that correct fitting of components is critical for favourable TKA outcomes. Traditionally, the IPFP has been removed in order to improve surgical exposure and to prevent interposition during baseplate implantation. ${ }^{12}$ Despite the significant evolution of TKA technology that no longer requires the resection of IPFP for better surgical access, IPFP is still partially or totally resected in around $88 \%$ of TKAs ${ }^{13}$ regardless of IPFP quality. Thus, evidence-based changes of practice towards preoperative IPFP evaluation and subsequent indication for resection or preservation are eagerly needed.

As for whether insufficient debridement of IPFP can cause anterior knee pain, our original cohort study was not able to provide an answer. We acknowledge that our findings, along with findings from our recent articles, ${ }^{45}$ were observational and there is not enough evidence to support preservation or resection of IPFP during TKA for patients with OA at very late stage. So far, there is no evidence to support the assumption that it is insufficient debridement, not the decision to debride the IPFP, that results in anterior knee pain.

In summary, our previous observational studies have suggested biphasic effects of IPFP on OA. In order to implement evidence-based changes of practice, well-designed randomised controlled trials are urgently required to test this hypothesis: preserve IPFP with normal quality and resect IPFP with pathological changes showing on MRI (signal intensity alterations) during TKA may have beneficial effects on patients' postsurgical outcomes.

\section{Zhaohua Zhu, ${ }^{1}$ Weiyu Han, ${ }^{2}$ Guangfeng Ruan, ${ }^{1,3}$ Shuang Zheng, ${ }^{4}$ Changhai Ding ${ }^{1,3,4}$ \\ ${ }^{1}$ Clinical Research Center, Zhujiang Hospital, Southern Medical University, Guangzhou, China \\ ${ }^{2}$ Department of Orthopedics, Zhujiang Hospital, Southern Medical University, Guangzhou, China \\ ${ }^{3}$ Department of Rheumatology and Immunology, Arthritis Research Institute, The First Affiliated Hospital of Anhui Medical University, Hefei, China \\ ${ }^{4}$ Menzies Institute for Medical Research, University of Tasmania, Hobart, Australia}

Correspondence to Dr Changhai Ding, Clinical Research Centre, Zhujiang Hospital, Southern Medical University, Guangzhou, China; Changhai.Ding@utas.edu.au

Handling editor Josef S Smolen

Contributors ZZ and CD had full access to all of the data in the study and take responsibility for the integrity and accuracy of the content. Study design: ZZ, WH, CD. Acquisition of data: ZZ, WH, GR, SZ and CD. Analysis and interpretation of data: ZZ, WH, GR, SZ and CD. Manuscript preparation: ZZ, WH, GR, SZ and CD.

Competing interests None declared.

Patient consent for publication Not required.

Provenance and peer review Commissioned; internally peer reviewed. (C) Author(s) (or their employer(s)) 2019. No commercial re-use. See rights and permissions. Published by BMJ.

\section{Check for updates}

To cite Zhu Z, Han W, Ruan G, et al. Ann Rheum Dis 2019;78:e64.

Received 11 June 2018

Accepted 11 June 2018

Published Online First 27 June 2018

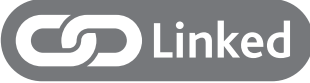

- http://dx.doi.org/10.1136/annrheumdis-2018-213684

Ann Rheum Dis 2019;78:e64. doi:10.1136/annrheumdis-2018-213701

\section{REFERENCES}

1 Ryan TJ. Infrapatellar fat pad resection during total knee arthroplasty: yet another reason for? Ann Rheum Dis 2019;78:e63.

2 Pan F, Han W, Wang $X$, et al. A longitudinal study of the association between infrapatellar fat pad maximal area and changes in knee symptoms and structure in older adults. Ann Rheum Dis 2015;74:1818-24.

3 Han W, Pan F, Liu Z, et al. Response to: 'The role of infrapatellar fat pad resection in total knee arthroplasty' by White et al. Ann Rheum Dis 2016;75:e67-E67.

4 Cai J, Xu J, Wang K, et al. Association between infrapatellar fat pad volume and knee structural changes in patients with knee osteoarthritis. J Rheumatol 2015;42:1878-84.

5 Han W, Aitken D, Zhu Z, et al. Signal intensity alteration in the infrapatellar fat pad at baseline for the prediction of knee symptoms and structure in older adults: a cohort study. Ann Rheum Dis 2016;75:1783-8.

6 Sekiya $\mathrm{H}$. Painful knee is not uncommon after total knee arthroplasty and can be treated by arthroscopic debridement. Open Orthop J 2017;11:1147-53.

7 Gwyn R, Kotwal RS, Holt MD, et al. Complete excision of the infrapatellar fat pad is associated with patellar tendon shortening after primary total knee arthroplasty. Eur J Orthop Surg Traumatol 2016;26:545-9.

8 Imren Y, Dedeoğlu SS, Çakar M, et al. Infrapatellar fat pad excision during total knee arthroplasty did not alter the patellar tendon length: a 5-year follow-up study. J Knee Surg 2017;30:479-83. 
9 Sellars H, Yewlett A, Trickett R, et al. Should we resect Hoffa's fat pad during total knee replacement? J Knee Surg 2017;30:894-7.

10 Yusuf E, Nelissen RG, loan-Facsinay A, et al. Association between weight or body mass index and hand osteoarthritis: a systematic review. Ann Rheum Dis 2010;69:761-5.

11 Chang J, Liao Z, Lu M, et al. Systemic and local adipose tissue in knee osteoarthritis. Osteoarthritis Cartilage 2018:864-71.
12 Van Beeck A, Clockaerts S, Somville J, et al. Does infrapatellar fat pad resection in total knee arthroplasty impair clinical outcome? A systematic review. Knee 2013;20:226-31.

13 Baker PN, van der Meulen JH, Lewsey J, et al. The role of pain and function in determining patient satisfaction after total knee replacement. Data from the National Joint Registry for England and Wales. J Bone Joint Surg $\mathrm{Br}$ 2007;89:893. 